\title{
Immunotherapy resistance of lung cancer
}

\author{
Xin Yu\#, Chaonan Han", Chunxia Su \\ Department of Oncology, Shanghai Pulmonary Hospital \& Thoracic Cancer Institute, Tongji University School of Medicine, \\ Shanghai 200433, China. \\ ${ }^{\#}$ Authors contributed equally.
}

Correspondence to: Chunxia Su, Department of Oncology, Shanghai Pulmonary Hospital \& Thoracic Cancer Institute, Tongji University School of Medicine, No. 507, Zheng Min Road, Shanghai 200433, China. E-mail: susu_mail@126.com

How to cite this article: Yu X, Han C, Su C. Immunotherapy resistance of lung cancer. Cancer Drug Resist 2022;5:114-28. https://dx.doi.org/10.20517/cdr.2021.101

Received: 14 Oct 2021 First Decision: 14 Oct 2021 Revised: 16 Dec 2021 Accepted: 30 Dec 2021 Published: 8 Feb 2022

Academic Editor: Godefridus J. (Frits) Peters Copy Editor: Yue-Yue Zhang Production Editor: Yue-Yue Zhang

\begin{abstract}
In recent years, immunotherapy has made remarkable breakthroughs and brought long-term survival benefits to lung cancer patients. However, a high percentage of patients do not respond to immunotherapy or their responses are transient, indicating the existence of immune resistance. Current studies show that the interactions between cancer cells and immune system are continuous and dynamic. A range of cancer cell-autonomous characteristics, tumor microenvironment factors, and host-related influences account for heterogenous responses. Furthermore, with the identification of new targets of immunotherapy and the development of immune-based combinations, we propose the response strategies to overcome resistance.
\end{abstract}

Keywords: Immunotherapy, resistance mechanisms, response strategies

\section{INTRODUCTION}

According to the latest cancer report of China, the morbidity and mortality of lung cancer are still the highest among all types of malignancies. Immune checkpoint inhibitors (ICIs) have dramatically changed the treatment landscape for advanced non-small cell lung cancer (NSCLC). In neoadjuvant therapy, ICIs combined with chemotherapy have increased the chances of cure for patients with early-stage NSCLC. In maintenance treatment, durvalumab significantly prolonged the disease-free survival time of NSCLC after concurrent radiotherapy and chemotherapy. The five-year survival rate of patients with advanced NSCLC 
who received ICI monotherapy increased from 5\% to $15 \%$. At the same time, ICIs combined with chemotherapy have also brought a giant breakthrough in the treatment of advanced small cell lung cancer (SCLC). However, the majority of patients treated with ICIs are either non-responders or eventually develop progressive disease ${ }^{[1,2]}$. Therefore, clarifying resistance mechanisms and proposing response strategies for immunotherapy are ongoing challenges that need to be coped with. The tumor is a heterogeneous and dynamic tissue, which continuously evolves in the attempt to overcome structural, metabolic, and immunologic hurdles. Thus, resistance mechanisms and pathways are intertwined. Generally, the resistance mechanism of immunotherapy can be classified into two categories: intrinsic mechanisms and extrinsic mechanisms. Intrinsic mechanisms refer to the genetic, transcriptional or functional profile of the tumor cells themselves, while the extrinsic mechanisms refer to the tumor microenvironment and factors other than the tumor itself. The changes of immunosuppressive cells, immunosuppressive cytokines, coinhibitory receptors, and costimulatory receptors in the tumor microenvironment can destroy the antitumor immune response, mediating resistance to immunotherapy. Host-related factors including physical status, previous comorbidities, distribution of intestinal flora, and use of antibiotics could also affect the efficacy of immunotherapy. This opinion article intends to discuss the currently known mechanisms of immune resistance and potential response strategies in lung cancer. The mechanisms and factors of immune resistance discussed in this opinion are summarized in Figure 1.

\section{RESISTANCE MECHANISMS OF IMMUNOTHERAPY IN LUNG CANCER}

\section{Intrinsic mechanisms and factors of resistance}

The intrinsic factors and mechanisms of resistance mainly refer to the expression or inhibition of specific genes and pathways of tumor cells, such as the activation of driver genes and the inactivation of suppressor genes, low tumor mutation burden (TMB), decreased tumor antigen presentation, and changes in the expression of programmed cell death ligand 1 (PD-L1) of tumor cells.

\section{Activation of driver genes}

Genomic or epigenetic alternations in tumor cells can mediate immune escape. Several driver genes have been identified in lung cancer, such as epidermal growth factor receptor (EGFR), anaplastic lymphoma kinase (ALK), and Kirsten rat sarcoma virus oncogene homolog (KRAS). They have all been confirmed to be related to the process of immune escape.

EGFR mutation is the most common type of mutation of NSCLC in Asians, with an incidence of 40.3\%$64.5 \%{ }^{[3]}$. Clinical trials showed that these patients do not respond well to immune checkpoint monotherapy ${ }^{[4-6]}$. There are several hypotheses for the inferior response of immunotherapy in this group of people. First, the immune microenvironment of this population belongs to the immune desert or immune excluded type with poor outcomes of immunotherapy ${ }^{[7,8]}$. After the activation of EGFR pathway, the ligand amphiregulin (AREG) could promote the production of regulatory $\mathrm{T}$ (Treg) cells to enhance the immunosuppressive effect through EGFR-glycogen synthase kinase-3 $\beta$ (GSK-3 $\beta$ )-forkhead box P3 (FOXP3) axis $^{[9]}$. Besides, the EGFR signaling pathway can also produce inhibitory cytokines, induce myeloid-derived suppressor cells (MDSC) and tumor-associated macrophages (TAM) proliferation, and inhibit CD8+ T Cell response $^{[10]}$. Second, the signal transducer and activator of the transcription 3 (STAT3) from the downstream of the signal pathway is upregulated, which in turn leads to the decreased expression of major histocompatibility complex I ${ }^{[11]}$. STAT3 also mediates the expression of vascular endothelial growth factor (VEGF), interleukin-6 (IL-6), and interleukin-10 (IL-10) and inhibits the differentiation and maturation of dendritic cells (DC). These changes will ultimately affect the presentation of antigens and the production of new antigens. Third, although most studies have demonstrated that EGFR will upregulate PD-L1, due to the decrease of new antigens and the increase of immunosuppressive cells, patients have not received durable 


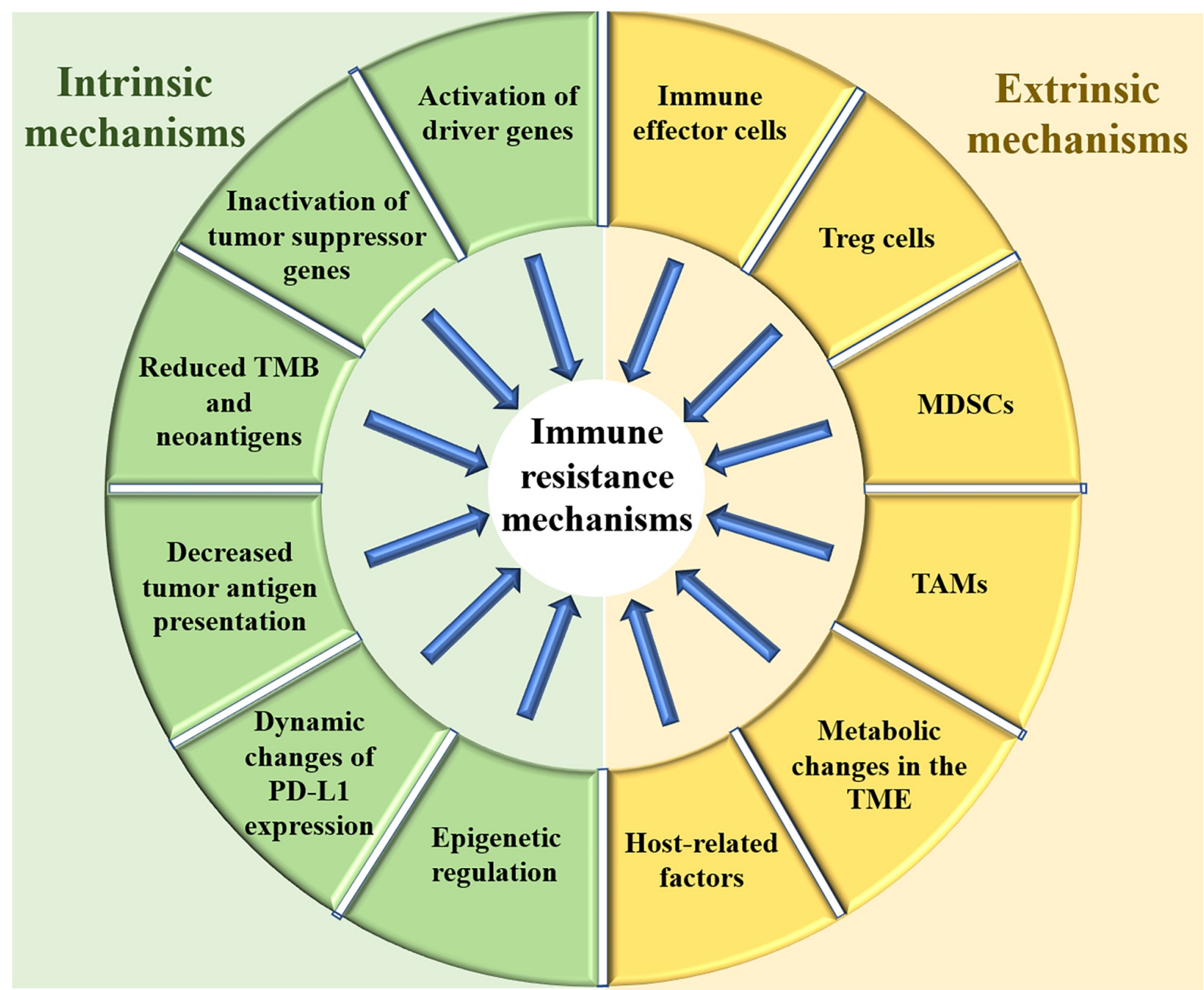

Figure 1. The intrinsic and extrinsic mechanisms and factors of immune resistance. TMB: Tumor mutation burden; PD-L1: programmed cell death ligand 1; MDSCs: myeloid-derived suppressor cells; TAMs: tumor-associated macrophages; TME: tumor microenvironment.

benefits from immune checkpoint monotherapy ${ }^{[10]}$.

Another common gene mutation in lung cancer is ALK fusion. ALK fusion can reduce the production of new antigens and increase the number of immunosuppressive cells through the PI3K-AKT and MEK-ERK pathways, resulting in poor efficacy of immune checkpoint monotherapy ${ }^{[12,13]}$. KRAS is another common driver gene in lung cancer. Studies showed that KRAS mutation could increase the expression of PD-L1 to promote immune escape by regulating the stability of the $3^{\prime} \mathrm{UTR}$ region of PD-L1 mRNA ${ }^{[14]}$. However, KRAS mutations are correlated with an inflammatory tumor microenvironment and tumor immunogenicity, resulting in superior patient response to PD-1/PD-L1 inhibitors. In a single-center retrospective study which included 25 cases of KRAS mutations and 47 wild-type cases, patients were administered with nivolumab \pm anti-CTLA-4 antibody. The median overall survival (OS) of the two groups was 18.1 and 8.1 months, respectively $(\mathrm{HR}=0.48, P=0.04)^{[15]}$. Mechanically, KRAS mutations induce the expression of neoantigens and change the expression a series of genes, such as cell cycle regulation, DNA replication, and DNA repair, but they have no obvious effect on activating immunosuppressive cells ${ }^{[16]}$. Several driver mutations of NSCLC and their chances to receive ICI therapy are summarized in Table 1. 
Table 1. Common driver mutations of NSCLC and their chances to receive ICI therapy

\begin{tabular}{|c|c|c|c|c|c|c|c|}
\hline \multicolumn{2}{|c|}{ EGFR mutation } & \multicolumn{2}{|c|}{ ALK mutation } & \multicolumn{3}{|c|}{ KRAS mutation } & \multirow{2}{*}{$\begin{array}{l}\text { Other mutations } \\
\mathrm{MET} / \mathrm{RET} / \mathrm{ROS} 1 / \mathrm{BRAF} / \mathrm{HER} 2\end{array}$} \\
\hline $\begin{array}{l}\mathrm{ICl} \\
\text { monotherapy }\end{array}$ & $\begin{array}{l}\mathrm{ICl} \text { combination } \\
\text { therapy }\end{array}$ & $\begin{array}{l}\mathrm{ICl} \\
\text { monotherapy }\end{array}$ & $\begin{array}{l}\mathrm{ICl} \text { combination } \\
\text { therapy }\end{array}$ & $\begin{array}{l}\text { Single } \\
\text { mutation }\end{array}$ & $\begin{array}{l}\mathrm{KP} \text { co- } \\
\text { mutations }\end{array}$ & $\begin{array}{l}\mathrm{KL} \mathrm{co-} \\
\text { mutations }\end{array}$ & \\
\hline$\times$ & $\sqrt{ }$ & $\times$ & $?$ & $\sqrt{ }$ & $\sqrt{ }$ & $x$ & $?$ \\
\hline $\begin{array}{l}\text { Not } \\
\text { recommended }\end{array}$ & $\begin{array}{l}\text { Consider using } \\
\mathrm{ICls} \text { in some } \\
\text { cases }\end{array}$ & $\begin{array}{l}\text { Not } \\
\text { recommended }\end{array}$ & $\begin{array}{l}\text { Consider using } \\
\mathrm{ICls} \text { in some } \\
\text { cases }\end{array}$ & $\begin{array}{l}\text { Probably } \\
\text { benefit } \\
\text { from ICls }\end{array}$ & $\begin{array}{l}\text { Probably } \\
\text { benefit from } \\
\text { ICls }\end{array}$ & $\begin{array}{l}\text { Not } \\
\text { recommended }\end{array}$ & $\begin{array}{l}\text { No large-scale trials and need } \\
\text { further research }\end{array}$ \\
\hline
\end{tabular}

NSCLC: Non-small cell lung cancer; ICIs: immune checkpoint inhibitors; EGFR: epidermal growth factor receptor; ALK: anaplastic lymphoma kinase; KRAS: Kirsten ratsarcoma viral oncogene homolog; KP: KRAS/TP53; KL: KRAS/STK11; MET: mesenchymal to epithelial transition factor; RET: ret proto-oncogene; ROS1: c-ros oncogene 1; BRAF: v-raf murine sarcoma viral oncogene homolog B; HER2: human epidermal growth factor receptor-2.

\section{Inactivation of tumor suppressor genes}

The inactivation of tumor suppressor genes, such as PTEN and STK11 genes, can also lead to poor outcomes of immunotherapy for lung cancer patients. In drug-resistant populations, the occurrence of the tumor suppressor PTEN mutations are significantly increased ${ }^{[17]}$. PTEN is a lipid phosphatase that inhibits the activity of the PI3K pathway. Current studies have shown that loss of PTEN expression is the most common way to activate the PI3K pathway in multiple cancers ${ }^{[18]}$. The PI3K pathway plays an indispensable role in tumor growth by regulating tumor proliferation and survival. Another study found a decreased expression of tumor-infiltrating lymphocytes (TILs) in patients with a high deletion rate of PTEN gene ${ }^{[19]}$. In addition, PTEN can upregulate the expression of VEGF and prevent tumor immune escape mediated by lymphocyte infiltration. STK11/LKB1 is another tumor suppressor gene. About 8\%-39\% of patients with NSCLC have STK1 1 gene mutation, which has the function of negatively regulating the rapamycin signal pathway. STK11 gene mutations are often accompanied by a decreased infiltration of cytotoxic CD8+ T lymphocytes, leading to the "cold" immune microenvironment ${ }^{[20]}$. Co-mutations of KRAS and STK11 (KL) or TP53 (KP) could also affect the efficacy of ICI therapy. Skoulidis et al. ${ }^{[21]}$ reported that patients with KRAS mutations and STK11/LKB1 alterations had reduced efficacy to immunotherapy in a cohort of 165 patients diagnosed with KRAS-mutated lung adenocarcinoma who had received PD-1/PD-L1 inhibitors treatment. In a mice model of KRAS mutation, the deletion of STK11 gene promotes the resistance of PD- 1 inhibitors monotherapy. Thus, KL tumors show resistance to PD-1 inhibitors, while KP tumors are more sensitive. Besides, the mutation rate of SMARCA4 gene in NSCLC is about $10 \%$, which is responsible for encoding the catalytic unit of the SWI/SNF chromatin remodeling complex. A study included 2690 NSCLC patients, of whom 211 patients carried SMARCA4 mutations ${ }^{[22]}$. Further analyses revealed that KRAS and SMARCA4 co-mutations are the most prevalent type. Among the NSCLC patients harboring KRAS mutations, patients with the co-occurrence of SMARCA4 mutation had a reduced objective response rate (ORR) (0.0\% vs. 23.5\%, $P=0.02)$, progression-free survival (PFS) (1.7 months vs. 4.1 months, $P<0.001)$, and median OS (4.0 months vs. 14.9 months, $P<0.001)$. In summary, mutations of driver genes and tumor suppressor genes can lead to changes in tumor neoantigens and can also alter the composition of the immune microenvironment, which is reflected in the heterogeneous efficacy of immunotherapy. Thus, it is necessary to interpret its impact on the efficacy of immunotherapy and the predictive value of drug resistance in clinical practice.

\section{Reduced TMB and neoantigens}

TMB is the number of substitution, insertion, and deletion mutations per megabase in the exon coding region of the evaluated gene. Yarchoan et al. ${ }^{[23]}$ analyzed the TMB of 27 tumor types and found a superior ORR of immune checkpoint monotherapy for tumor types with high TMB. NSCLC and melanoma have relatively higher mutation loads and immunogenicity, and they are more sensitive to ICI therapy. On the contrary, pancreatic cancer, prostate cancer, and thyroid carcinoma have lower mutation loads and exhibit 
lower immune responses ${ }^{[23-25]}$. Tumor neoantigens refer to antigens that are not expressed in normal tissues but only exist in tumor tissues, which are usually highly immunogenic. Several studies have shown that increased TMB, DNA mismatch repair gene deletion, and high genomic microsatellite instability can increase tumor antigen expression and improve immune efficacy ${ }^{[24,26]}$. Anagnostou et al.$^{[27]}$ found that the neoantigen load of NSCLC patients treated with pembrolizumab with sustained clinical benefit was significantly higher than that of patients with non-sustained benefit. The study also found that 7-18 mutation-related neoantigens that can produce anti-cancer responses were lost and replaced by more complicated new mutations before drug resistance. The proportion of new mutations involved in encoding tumor antigens had decreased ( $8 \% v$ s. 19\%) and the clonality of T cell receptors (TCR) was changed, leading to the acquired drug resistance.

\section{Decreased tumor antigen presentation}

The major histocompatibility complex (MHC) is a key molecule involved in the immune response, participating in the processing and presentation of antigens, thereby activating $\mathrm{T}$ cells and mediating the immune response. $\beta 2$-microglobulin $(\beta 2-\mathrm{GM})$ is one of the important proteins that compose the heavy chain of MHC class I molecules, which is responsible for folding and transporting MHC class I molecules to the cell surface ${ }^{[28]}$. Studies confirmed that $B 2 M$ gene mutations can lead to the loss of MHC class I molecules on the cell surface, which in turn promotes the recognition dysfunction of CD8+ T cells and induces immune resistance in NSCLC ${ }^{[29]}$. In addition, studies found that defective expression of MHC class II molecules in tumor cells and infiltrating lymphocytes cannot effectively activate $\mathrm{T}$ helper cells. The low antigen presentation reduces the immunogenicity of lung cancer, while weak immunogenic tumors can escape the surveillance of the immune system and promote immunotherapy resistance in lung cancer ${ }^{[30]}$.

\section{Dynamic changes of PD-L1 expression}

Tumor cells with high expression of PD-L1 can also mediate immune escape. PD-L1 could transmit negative signals and induce $\mathrm{T}$ cell apoptosis or dysfunction when combined with its receptor, thereby evading the immune system. In patients with acquired resistance, studies have found that interferon- $\gamma$ $(\mathrm{IFN}-\gamma)$ secreted by both TILs and tumor antigen-specific T cells can mediate the upregulation of PD-L1 expression after tumor antigen recognition. In addition, there are many ways for tumors to upregulate the expression of PD-L1. For example, the currently known PTEN deletion ${ }^{[18]}$, PI3K, or AKT mutations ${ }^{[31]} \mathrm{can}$ induce the expression of PD-L1 in tumor cells to escape from immune system. However, clinical trials including KEYNOTE-024 and KEYNOTE-208 have demonstrated that the high PD-L1 expression of tumor cells were more sensitive to immunotherapy ${ }^{[2,33]}$. Chinese Society of Clinical Oncology guidelines and The National Comprehensive Cancer Network guidelines also indicate that the expression level of PD-L1 can be used as one of the predictors of the efficacy of immunotherapy. These studies suggest that the functions of PD-L1 may be diverse, rather than simply categorized as "promoting" or "inhibiting". A recent study confirmed that deacetylation-dependent nuclear translocation of PD-L1 promotes immune escape and upregulates the immune checkpoint genes such as PD-L2 and VISTA of tumor cells, thereby mediating resistance to $\mathrm{PD}-1 / \mathrm{PD}-\mathrm{L} 1$ inhibitors $^{[34]}$. In addition, nuclear PD-L1 can also induce immune responserelated gene expression, including type I and type II IFN signaling pathways, nuclear factor kappa-B (NF- $\mathrm{kB}$ ) signaling pathways, and antigen presentation pathways. These results indicate the dual role of PDL1, which participates in both drug resistance and immune response pathways.

\section{Epigenetic regulation}

Epigenetics is the study of heritable changes in gene expression caused by mechanisms other than changes in the nucleotide sequence of the gene, including DNA methylation, histone modification, nucleosome remodeling, and non-coding RNA expression. Epigenetic changes can affect the expression of immune 
checkpoints, disrupt the process of antigen presentation, and inhibit the migration of $\mathrm{T}$ cells to the tumor microenvironment and the activation of $\mathrm{T}$ cells ${ }^{[35]}$. In addition, miRNA is also involved in the above process. Studies have shown that miR-214, miR-126, and miR-568 could promote the development and function of Treg cells and lead to $\mathrm{T}$ cell exhaustion ${ }^{[35]}$.

\section{Extrinsic mechanisms and factors of resistance}

The extrinsic mechanisms and factors of resistance mainly refer to the tumor microenvironment (TME). The TME is the internal environment for the emergence and growth of tumor cells. Immunologists have tried to find ways to positively affect the immune response, such as the usage of cytokines, adoptive immunotherapy, and other treatments, but the results are not satisfactory. Recently, researchers are focusing on the balance between the "positive factors", such as immune effector cells, and "negative factors", such as Treg cells and MDSC in TME to enhance the efficacy of immunotherapy.

\section{Immune effector cells}

The lower infiltration and exhaustion of immune effector cells lead to immune suppression and mediate immune escape. Based on the distribution of CD8+ T cells, the immune phenotypes are divided into three types: immune inflamed, immune excluded, and immune desert. In a phase II clinical trial of durvalumab combined with olaparib in SCLC, 14 evaluable patients were included, of whom nine were immune excluded, three were immune-inflamed, and two were immune desert type. They study found that immunotherapy may play a role in the TME infiltrated by CD8+ T cells ${ }^{[36]}$. Guo et al.$^{[37]}$ analyzed the singlecell sequencing of 12,346 T cells in the peripheral blood, cancer tissue, and adjacent tissues from 14 patients with NSCLC, and they found that infiltrating T cells were mainly comprised of three subgroups. In addition to tumor-infiltrating CD8+ T cells undergoing exhaustion, two other clusters of cells exhibited states preceding exhaustion, and a high ratio of "pre-exhausted" to exhausted T cells was associated with better prognosis of lung adenocarcinoma. NK cells are not restricted by MHC or antibodies and can directly release perforin and TNF to exert their immune functions. The dysfunction of NK cells could mediate immune escape through such mechanisms as overexpression of inhibitory receptors and impaired production of cytokines. Trefny et al. ${ }^{[38]}$ analyzed 35 patients with NSCLC who were treated with nivolumab and found a correlation between immunoglobulin-like receptor gene KIR3DS1 expressed by NK cells and therapeutic efficacy. Mutations of KIR3DS1 gene could lead to resistance to immunotherapy. In addition, several studies have revealed the relationship between the immune response of B cells and tertiary lymphoid structures (TLS). Compared with non-responders, responders have significantly higher B cell-related gene expression levels before ICI treatment. The density of CD20-positive B cells and TLS in the tissues and the ratio of TLS to tumor area in patients who respond to immunotherapy were significantly higher ${ }^{[39-41]}$. In general, these studies have shown the role of immune effector cells in regulating anti-tumor immunity and immunotherapy efficacy.

\section{Treg cells}

Treg cells are a type of $\mathrm{T}$ cell group with immunosuppressive function, which can inhibit the activation and proliferation of CD4+ and CD8+ T cells and the functions of naive and memory T cells. Treg cells play an indispensable role in maintaining the balance of autoimmunity and can effectively weaken the immunity of autoantigens. However, they are also "utilized" by tumors cells that could express autoantigens to avoid immune surveillance ${ }^{[42]}$. Elevated Treg levels were found in lung, breast, and pancreatic cancers ${ }^{[43]}$, while the Treg levels were significantly reduced after surgical removal of the tumor ${ }^{[44]}$. Animal experiments ${ }^{[45]}$ have proved that the depletion of CD25+ Treg cells could effectively improve the anti-tumor immune response of mice. Thus, it is believed that the elimination of Treg cells should be an essential part of the treatment. Several chemotherapeutic drugs are known to interfere with the function of Treg cells non-specifically, including cyclophosphamide, gemcitabine, methotrexate, and thalidomide ${ }^{[46]}$. Drugs for Tregs targeting 
CD25 and CTLA-4 and eliminating tumor-related Treg cells include the well-known CTLA-4 antibodies ipilimumab and tremelimumab. In addition, the anti-CD25 antibody daclizumab ${ }^{[4]}$, the anti-OX40 (CD134) monoclonal antibody ${ }^{[48]}$, and the chemokine receptor $8^{[49]}$ are in phase I clinical trials, which bring hopes for the treatment of cancer patients.

\section{MDSCs}

MDSC is a type of cell population with immunosuppressive function, which can mediate immune escape and immune tolerance through a variety of ways ${ }^{[50]}$. In addition to secreting molecules such as TGF- $\beta$ and IL-10 that directly inhibit T effector cells, MDSCs can also induce the proliferation of other immunosuppressive cells. Studies have shown that MDSCs could promote the production of FOXP3+ Treg cells by secreting IFN- $\gamma$ and IL-10 ${ }^{[5,52]}$. MDSCs can also exert immunosuppressive effects by blocking lymphocyte homing and regulating enzymes required for adenosine metabolism. Kim et al.$^{[53]}$ found that the MDSC level of tumor-bearing mice that are resistant to ICIs increased by 5-7 times compared with nontumor-bearing mice. They also found that a high level of PI3K $\gamma$ in MDSCs can promote the production of inflammatory mediators and immunosuppressive factors. When combining PI3K inhibitors with PD-1 /CTLA-4 inhibitors, the MDSC level of tumor-bearing mice decreased significantly and was comparable to the control group. De Henau et al. ${ }^{[54]}$ verified that PI3K $\gamma$ inhibitors could remodel the immune microenvironment and prevent tumor growth. High expression of indoleamine 2,3-dioxygenase (IDO) was found on MDSCs in immune-resistant lung cancers. Previous studies showed that the expression level of IDO was positively correlated with clinical stages ${ }^{[55]}$. The application of IDO inhibitor INCB023843 downregulates the expression of IDO and increases the infiltration of CD8+ T cells, thereby reactivating the $\mathrm{T}$ cell anti-tumor response ${ }^{[56]}$. These studies reveal the potential value of suppressing MDSCs and its combination with immune checkpoint inhibitors.

\section{TAMs}

Circulating monocytes and macrophages are recruited into the tumor site and change the tumor microenvironment in the process of tumor progression. The phenotypes of macrophages could be transformed with the changes of signals produced by tumor and stromal cells. Macrophages could be divided into two categories based on their functions. M1 type macrophages are related to inflammatory response, pathogen elimination, and anti-tumor immunity, while M2 type macrophages have tumorpromoting properties. TAMs are very similar to M2 type macrophages ${ }^{[57]}$. Studies of multiple tumor types including breast cancer ${ }^{[58]}$, ovarian cancer $^{[59]}$, and NSCLC $^{[60]}$ have shown that the aggregation of macrophages is positively correlated with CCL2 levels. In addition to CCL2, other chemokines such as CCL3, CCL4, CCL5, and cytokines are also involved in the recruitment of macrophages ${ }^{[6,62]}$. TAMs inhibit the function of $\mathrm{T}$ cells by weakening the ability of antigen presentation and releasing immunosuppressive factors such as IL-10 and TGF- $\beta$. Studies have shown that the inhibition of the recruitment of macrophages through the regulation of chemotactic agents is effective. For example, bindarit is used to inhibit CCL2 ${ }^{[63]}$, and monoclonal antibodies that block VEGFR2 reduce macrophage infiltration and tumor growth ${ }^{[64]}$. CSF-1R blockade can also reduce the number of TAMs and activate T cells in TME ${ }^{[65]}$. These preclinical trials have provided a basis for reversing the drug resistance raised by TAMs.

\section{Metabolic changes in the TME}

Metabolic changes in the TME can reduce immune effects by producing immunosuppressive metabolites to inhibit immune cell infiltration ${ }^{[66]}$. For example, some tumors use glutamine as an energy source, and the metabolically decomposed ammonia can activate autophagy in neighboring immune cells. Arginine metabolism plays an important role in the activation of $\mathrm{T}$ cells and the regulation of immune response. The immunoregulatory cells expressing arginase $1(\mathrm{ARG} 1)$ in TME degrade arginine and restrict its use by $\mathrm{T}$ 
cells. Therefore, inhibition of arginase in TME can enhance the efficacy of ICIs. A clinical trial of the combination of ARG1 inhibitor INCB001158 and nivolumab is ongoing. Besides, the rate of fatty acid synthesis is increased to produce cell membrane phospholipids and signal molecules in tumor cells, and targeting these metabolic pathways is a promising way to enhance anti-tumor immunity. In recent years, many studies have focused on the metabolic process of the TME, providing new perspectives for overcoming immune resistance.

\section{Host-related factors}

The immune system function degenerates with age, and the number and function of antigen-presenting cells and lymphocytes are also decreased ${ }^{[67]}$. A study found that elderly patients with melanoma responded better to immunotherapy than younger people ${ }^{[68]}$. A meta-analysis including 11,351 cases with advanced or metastatic cancer found that male patients had superior efficacy of immunotherapy ${ }^{[6]}$. Compared with the control group, the overall survival HR of male and female patients treated with ICIs was 0.72 and 0.86 , respectively $(P=0.0019)$. In addition, studies have suggested that weight can also affect the efficacy of immunotherapy. A study conducted a pooled analysis of four studies of OAK, POPLAR, BIRCH, and FIR, reporting a correlation between the overall survival of atezolizumab in patients with advanced NSCLC and body mass index ${ }^{[7]}$. The results show that obesity was associated with a significant increase in the OS of patients receiving PD-L1 inhibitors treatment, and the risk of death in obese patients was reduced by $64 \%$ $(\mathrm{HR}=0.36,95 \% \mathrm{CI}: 0.21-0.62)$. Smoking is also a crucial factor. The average frequency of gene mutations in smokers is more than 10 times higher than that of non-smokers ${ }^{[7]}$. A recent study reported that benzopyrene in cigarettes can induce an increase in PD-L1 level, which further explains why smoking patients respond better to immunotherapy ${ }^{[72]}$. In addition, studies have shown that gut microbiota have a significant impact on the efficacy of immunotherapy ${ }^{[73]}$. There is evidence of the importance of the intestinal microbiota in the response to chemotherapy and immunotherapy and how their alteration and the concomitant use of antibiotics inhibit the benefit of ICIs in advanced cancer, decreasing OS and PFS in NSCLC. With respect to microbiota composition, the relative abundance of Akkermansia muciniphila appears to significantly affect the clinical response to anti-PD-1/PD-L1 therapy in NSCLC and renal cell carcinoma $^{[74]}$. Another study demonstrated that a greater diversity of the gut microbiome is related to favorable responses to ICI therapy in NSCLC ${ }^{[75]}$. Among the different immune cells, the microbiota have been shown to be associated with the development of effector cells of the immune system, such as Th1, Th2, Th17, and Treg cells $s^{[6-78]}$. However, in the case of SCLC, there is little evidence that supports the role of the microbiome as an immune resistance mechanism. Thus, host-related factors could also affect the efficacy of immunotherapy.

\section{IMMUNOTHERAPY-BASED COMBINATIONS TO COPE WITH RESISTANCE}

There are no standard treatments after resistance of immunotherapy. At present, the most promising and effective treatment methods are immunotherapy combinations that aim to convert "cold" tumors with lower immune response into "hot" tumors with better response. A variety of combined treatment strategies, such as immunotherapy combined with targeted therapy, chemotherapy, and radiotherapy can modulate the immune response at different stages and overcome drug resistance. Clinical trials of immunotherapybased combinations related to lung cancer patients are summarized in Table 2.

\section{Dual immunotherapy}

Anti-tumor immune response involves antigen recognition, presentation, and activation of immune cells. Currently, many studies focus on the immune checkpoint inhibitors targeting PD-1, PD-L1, lymphocyte activating gene 3 (LAG-3), T cell immunoglobulin domain and mucin domain-3 (TIM-3), and T cell immunoglobulin and ITIM domain protein (TIGIT). The combined application of these immune 
Table 2. Clinical trials of immunotherapy-based combinations related to lung cancer patients

\begin{tabular}{|c|c|c|c|}
\hline $\begin{array}{l}\text { Immunotherapy-based } \\
\text { combinations }\end{array}$ & Combination strategies & Drugs & Clinical trials \\
\hline \multirow[t]{7}{*}{ Dual immunotherapy } & Anti-PD-1 + Anti-CTLA-4 & Nivolumab + Ipilimumab & $\begin{array}{l}\text { Checkmate 227 } \\
\text { (NCTO2477826) }\end{array}$ \\
\hline & \multirow[t]{3}{*}{ Anti-PD-1 + Anti-LAG-3 } & Nivolumab + MK-4280 & $\begin{array}{l}\text { Keynote } 495 \\
\text { (NCT03516981) }\end{array}$ \\
\hline & & Nivolumab + Relatimab & NCT03607890 \\
\hline & & Pembrolizumab + IMP321 & NCT03625323 \\
\hline & \multirow[t]{2}{*}{$\begin{array}{l}\text { Anti-PD-1/PD-L1 + Anti- } \\
\text { TIGIT }\end{array}$} & Atezolizumab + Tiragolumab & $\begin{array}{l}\text { CITYSCAPE } \\
\text { (NCT03563716) }\end{array}$ \\
\hline & & Pembrolizumab + MK-7684 & NCT02964013 \\
\hline & Anti-PD-L1 + Anti-TIM-3 & LY3300054 + LY3321367 & NCT03099109 \\
\hline \multirow[t]{5}{*}{ ICls combined with CT } & \multirow[t]{5}{*}{ Anti-PD-1/PD-L1 + CT } & \multirow[t]{2}{*}{ Pembrolizumab + Pemetrexed/Platinum } & $\begin{array}{l}\text { Keynote } 189 \\
\text { (NCT02578680) }\end{array}$ \\
\hline & & & $\begin{array}{l}\text { Keynote } 021 \\
\text { (NCT02039674) }\end{array}$ \\
\hline & & \multirow[t]{2}{*}{ Atezolizumab + Paclitaxel/Carboplatin } & $\begin{array}{l}\text { IMpower } 130 \\
\text { (NCT02367781) }\end{array}$ \\
\hline & & & $\begin{array}{l}\text { IMpower } 131 \\
\text { (NCT02266949) }\end{array}$ \\
\hline & & Atezolizumab + Etoposide/Carboplatin & $\begin{array}{l}\text { IMpower } 133 \\
\text { (NCT02763579) }\end{array}$ \\
\hline \multirow[t]{3}{*}{$\begin{array}{l}\text { ICls combined with targeted } \\
\text { therapy }\end{array}$} & $\begin{array}{l}\text { Anti-PD-L1 + Anti-VEGF + } \\
\text { CT }\end{array}$ & $\begin{array}{l}\text { Atezolizumab + Bevacizumab + } \\
\text { Paclitaxel/Carboplatin }\end{array}$ & $\begin{array}{l}\text { IMpower } 150 \\
\text { (NCT02366143) }\end{array}$ \\
\hline & Anti-PD-L1 + Anti-RTK & Nivolumab + Sitravatinib & NCT02954991 \\
\hline & $\begin{array}{l}\text { Anti-PD-L1 + Multitarget } \\
\text { inhibitor }\end{array}$ & Atezolizumab + Cabozantinib & $\begin{array}{l}\text { COSMIC-021 } \\
(\text { NCT03170960) }\end{array}$ \\
\hline \multirow[t]{2}{*}{ ICls combined with RT } & \multicolumn{2}{|c|}{ Durvalumab after concurrent $\mathrm{RT}+\mathrm{CT}$} & PACIFIC (NCT02125461) \\
\hline & \multicolumn{2}{|l|}{ Pembrolizumab + SBRT } & $\begin{array}{l}\text { PEMBRO-RT } \\
\text { (NCT02492568) }\end{array}$ \\
\hline \multirow[t]{2}{*}{ Individualized immunotherapy } & Anti-PD-1 + Vaccine & Nivolumab + NEO-PV-01 & NCT02897765 \\
\hline & Anti-PD-1 + MDM2 inhibitor & Pembrolizumab + APG-115 & NCT03611868 \\
\hline
\end{tabular}

ICIs: Immune checkpoint inhibitors; PD-1: programmed cell death 1; PD-L1: programmed cell death ligand 1; CTLA-4: cytotoxic T lymphocyteassociated antigen-4; LAG-3: lymphocyte activation gene-3; TIGIT: T cell immunoglobulin and ITIM domains; TIM-3: T cell immunoglobulin-3; CT: chemotherapy; RTK: receptor tyrosine kinases; RT: radiotherapy; SBRT: stereotactic body radiation therapy; MDM2: mouse double minute 2 homolog.

checkpoint inhibitors could exert synergistic effects. The most common strategy is PD-1 inhibitor combined with anti-CTLA-4 antibody. Both CheckMate 227 and CheckMate 032 studies have confirmed that the efficacy of dual-immunotherapy is better than that of single-agent therapy ${ }^{[79,80]}$. LAG-3 is expressed on the activated CD4+, CD8+ T cells, NK cells, and Treg cells, which can bind to its ligand fibrinogen-like protein-1 (FGL1) to inhibit T cell function. The trial of LAG-3 antibody MK-4280 combined with pembrolizumab in advanced NSCLC (KEYNOTE-495/NCT03516981) recruited 33 patients with solid tumors who have failed previous treatments. The DCR of the monotherapy group and the combination treatment group was $17 \%$ and $40 \%$, respectively. TIGIT is a specific negative regulator of CD226 costimulatory receptor and plays an important role in immunosuppression. The CITYSCAPE study enrolled 135 patients with PD-L1-positive treatment-naïve NSCLC who were randomly assigned to TIGIT antibody tiragolumab combined with atezolizumab (TA group) or placebo combined with atezolizumab (PA group). The results show that, in the intention-to-treat population, the ORR and PFS of the TA group was increased compared with the PA group (31.3\% vs. 16.2\%; 5.4 months vs. 3.6 months), and the risk of disease progression was reduced by $43 \%^{[81]}$. In addition, the phase II clinical trial of relatimab combined with nivolumab in the treatment of solid tumors (NCT03607890) and the phase II clinical trial of IMP321 
combined with pembrolizumab in the treatment of patients with advanced NSCLC (NCT03625323) are both underway. The phase I clinical trial of pembrolizumab combined with TIGIT antibody in the treatment of patients with metastatic solid tumors (NCT02964013) and the phase I trial of TIM-3 inhibitor combined with PD-L1 antibody in the treatment of advanced relapsed/refractory solid tumors (NCT03099109) are ongoing.

\section{Immunotherapy combined with chemotherapy}

Immunotherapy combined with chemotherapy can not only increase the cross-presentation of antigens by $\mathrm{DCs}^{[82]}$ but also eliminate the immunosuppressive components of the $\mathrm{TME}^{[83]}$, such as Treg cells, MDSCs, and immunosuppressive cytokines. The KEYNOTE-189 study showed that the efficacy of pembrolizumab combined with chemotherapy in patients with advanced NSCLC in first line is superior to chemotherapy alone ${ }^{[84]}$. The OS of the two groups was 22.0 and 10.7 months and the PFS was 9.0 and 4.9 months, respectively. In addition, the CheckMate 012, KEYNOTE-021, IMpower130, and IMpower131 studies have shown that immunotherapy combined with chemotherapy has a greater survival benefit than chemotherapy along despite using different checkpoint inhibitors. Based on these studies, the U.S. Food and Drug Administration has approved nivolumab and pembrolizumab combined with platinum-based chemotherapy for EGFR/ALK wild-type NSCLC. Besides, results from the double-blind, randomized phase III study IMpower 133 show that, compared with chemotherapy alone, atezolizumab combined with chemotherapy in the first-line treatment of extensive-stage SCLC achieved OS benefit, which represents a breakthrough in the treatment of SCLC ${ }^{[85]}$.

\section{Immunotherapy combined with targeted therapy}

Targeted therapy can increase tumor antigens and play a synergistic effect with immunotherapy in multiple aspects. Anti-angiogenic agents can promote the normalization of blood vessels, enhance immune effector cells to infiltrate the tumor, and reduce the activity of immunosuppressive cells. The IMpower150 study evaluated atezolizumab combined with bevacizumab and chemotherapy in the first-line treatment of nonsquamous NSCLC patients, including EGFR and ALK mutant populations, and the results confirm that patients in ABCP group have significant survival benefits ${ }^{[86]}$. Cohort 7 of the COSMIC-021 study included 30 advanced NSCLC patients with negative driver genes who had previously been treated with immune checkpoint inhibitors. The results show that the ORR was $23 \%$, DCR was $83 \%$, and the median duration of response was 5.6 months $^{[87]}$. A phase I randomized clinical study explored the efficacy of nivolumab combined with erlotinib in the treatment of patients with EGFR-mutant advanced NSCLC. Twenty EGFRTKI resistant cases and one EGFR-naive case were included. The results show that the median PFS was 5.1 months ${ }^{[88]}$. In addition, the combination of novel targeted agents with immunotherapy has also shown great prospects. Nivolumab combined with the small molecule inhibitor sitravatinib in the treatment of patients with advanced non-squamous NSCLC who are resistant to immunotherapy obtained an ORR of $32.14 \%$, a median PFS of 6.8 months, and a median OS of 15.1 month $s^{[89]}$. Pembrolizumab combined with JAK1 inhibitor itacitinib in the treatment of NSCLC patients with PD-L1 expression > 50\% reported an ORR of $66.7 \%$.

\section{Immunotherapy combined with radiotherapy}

The PACIFIC study included 713 patients with locally advanced unresectable NSCLC. The results show that the median PFS of the durvalumab treatment group after concurrent radiotherapy and chemotherapy was 17.2 months, and the median OS was also significantly improved compared with control group ${ }^{[00]}$. The PEMBRO-RT study explored the efficacy of radiotherapy and concurrent immunotherapy. Patients were treated with pembrolizumab seven days after receiving stereotactic body radiotherapy, while the control group only used immunotherapy. The ORR of the two groups was $36 \%$ and $18 \%$ and the median OS was 15.9 and 7.6 months, respectively ${ }^{[91]}$. In addition, a retrospective study included 26 NSCLC patients with 
acquired resistance of immunotherapy. In total, 15 patients were administered with local treatment without systemic treatment, and 11 of them continued to receive immunotherapy after local treatment. The twoyear survival rate was $92 \%$, and the median OS has not yet been reached ${ }^{[92]}$. Similarly, a retrospective study including 189 patients with acquired resistance showed that local treatment can significantly improve survival benefits ${ }^{[93]}$.

Other combination strategies are also being explored in immunotherapy-resistant populations. The VARGADO study included 57 patients with advanced NSCLC after progression with second-line immunotherapy. Patients were treated with the VEGF inhibitor nintedanib combined with docetaxel. The results show that ORR and DCR are 50\% and 85\%, respectively. The median PFS was 6.5 months and the median OS was 12.4 months ${ }^{[04]}$. Although the ideas for overcoming immunotherapy resistance are various, there exist some problems that cannot be ignored while conducting clinical trials. Firstly, adequate preclinical evaluation should be carried out when the clinical trial is designed, and the adverse effects should be monitored. For example, in the PACIFIC study, the incidence of radiation pneumonitis in the immunotherapy group was increased (33.9\% vs. $24.8 \%)$, and $15.4 \%$ of patients discontinued treatment due to adverse events. In the TATTON study, the incidence of interstitial pneumonitis in NSCLC patients receiving durvalumab combined with osimertinib was significantly increased; thus, the treatment group was promptly interrupted in the corresponding phase III clinical trial (CAURAL study). Novel treatment strategies such as immunotherapy combined with molecular inhibitors need to further consider the potential safety profiles raised by the superposition of different drugs. Secondly, the rationality of the combination strategy is supposed to be evaluated. The selected treatment drugs and immune checkpoint inhibitors should have synergistic effects in the immune feedback loop.

\section{Individualized immunotherapy}

Given the characteristics of the tumor immune microenvironment, developing drugs for tumor cell neoantigen production and presentation, $\mathrm{T}$ cell activation, and immunosuppressive microenvironment, such as oncolytic viruses, tumor vaccines, and adoptive immune cell therapy (TIL, CAR-T, TCR-T, CAR$\mathrm{NK}$, etc.), which are based on the characteristics of individualized immune microenvironments represent promising directions for overcoming immunotherapy resistance. The NT-001 study is a phase Ib clinical trial that intends to explore the efficacy of PD-1 inhibitors combined with vaccines for advanced or metastatic melanoma, smoking-related NSCLC, and bladder cancer. In total, 82 patients were enrolled. ORR for metastatic NSCLC patients reached $45.5 \%$. MDM2 (mouse double minute 2 homolog) is one of the most important inhibitors of p53; it degrades the p53 protein and weakens its tumor suppressor effect. APG-115 is a second-generation MDM2 inhibitor that can block the MDM2-p53 interaction, thereby restoring the transcriptional regulatory function of the 53 protein, promoting cell apoptosis, and restoring tumor suppressor activity. The phase Ib clinical trial results of APG-115 combined with pembrolizumab in the treatment of metastatic melanoma or advanced solid tumors confirm that the combination of the two drugs is well tolerated, with an ORR of $16.7 \%$ and a DCR of $55.5 \%$. With the continuous in-depth study of immunotherapy mechanisms, individualized immunotherapy targets may be a new strategy with good development prospects.

\section{CONCLUSIONS AND FUTURE DIRECTIONS}

Compared with traditional chemotherapy and targeted therapy, immunotherapy has its unique advantages and brings a new light to lung cancer treatment. With the clinical application of immunotherapy, anti-PD-1 /PD-L1 monotherapy resistance has become an unavoidable phenomenon. The ongoing research on the mechanisms of immune resistance provides new ideas for the selection of patient populations and response strategies for reversing immune resistance. Immunotherapy-based combinations are becoming one of the 
most promising strategies that convert non-responders to responders. In the future, studies on biomarker identifications are required to predict the efficacy and prognosis of immunotherapy. Further exploration is needed to predict immune resistance and the timing of restarting immunotherapy.

\section{DECLARATIONS}

\section{Authors' contributions}

Manuscript writing: Yu X, Han C

Manuscript revision: $\mathrm{Su} \mathrm{C}$

Read and approved the final manuscript: Yu X, Han C, Su C

\section{Availability of data and materials}

Not applicable.

\section{Financial support and sponsorship}

This work was supported by National Natural Science Foundation of China (grant number: 81874036, 82072568), Science and Technology Commission of Shanghai Municipality (19411971100) and Shanghai Shenkang Hospital Development Center (SHDC12020110).

\section{Conflicts of interest}

All authors declared that there are no conflicts of interest.

\section{Ethical approval and consent to participate}

Not applicable.

\section{Consent for publication}

Not applicable.

\section{Copyright}

(c) The Author(s) 2022.

\section{REFERENCES}

1. Walsh RJ, Soo RA. Resistance to immune checkpoint inhibitors in non-small cell lung cancer: biomarkers and therapeutic strategies. Ther Adv Med Oncol 2020;12:1758835920937902. DOI PubMed PMC

2. Boyero L, Sánchez-Gastaldo A, Alonso M, Noguera-Uclés JF, Molina-Pinelo S, Bernabé-Caro R. Primary and acquired resistance to immunotherapy in lung cancer: unveiling the mechanisms underlying of immune checkpoint blockade therapy. Cancers (Basel) 2020;12:3729. DOI PubMed PMC

3. Dearden S, Stevens J, Wu YL, Blowers D. Mutation incidence and coincidence in non small-cell lung cancer: meta-analyses by ethnicity and histology (mutMap). Ann Oncol 2013;24:2371-6. DOI PubMed PMC

4. Borghaei H, Paz-Ares L, Horn L, et al. Nivolumab versus docetaxel in advanced nonsquamous non-small-cell lung cancer. $N$ Engl $J$ Med 2015;373:1627-39. DOI PubMed PMC

5. Herbst RS, Baas P, Kim D, et al. Pembrolizumab versus docetaxel for previously treated, PD-L1-positive, advanced non-small-cell lung cancer (KEYNOTE-010): a randomised controlled trial. Lancet 2016;387:1540-50. DOI PubMed

6. Rittmeyer A, Barlesi F, Waterkamp D, et al. Atezolizumab versus docetaxel in patients with previously treated non-small-cell lung cancer (OAK): a phase 3, open-label, multicentre randomised controlled trial. Lancet 2017;389:255-65. DOI PubMed PMC

7. Chen DS, Mellman I. Elements of cancer immunity and the cancer-immune set point. Nature 2017;541:321-30. DOI PubMed

8. Dong ZY, Zhang JT, Liu SY, et al. EGFR mutation correlates with uninflamed phenotype and weak immunogenicity, causing impaired response to PD-1 blockade in non-small cell lung cancer. Oncoimmunology 2017;6:e1356145. DOI PubMed PMC

9. Wang S, Zhang Y, Wang Y, et al. Amphiregulin confers regulatory T cell suppressive function and tumor invasion via the EGFR/GSK-3ß/Foxp3 axis. J Biol Chem 2016;291:21085-95. DOI PubMed PMC

10. Chen N, Fang W, Zhan J, et al. Upregulation of PD-L1 by EGFR activation mediates the immune escape in EGFR-driven NSCLC: implication for optional immune targeted therapy for NSCLC patients with EGFR mutation. J Thorac Oncol 2015;10:910-23. DOI PubMed

11. Zhang N, Zeng Y, Du W, et al. The EGFR pathway is involved in the regulation of PD-L1 expression via the IL-6/JAK/STAT3 signaling pathway in EGFR-mutated non-small cell lung cancer. Int J Oncol 2016;49:1360-8. DOI PubMed 
12. Gainor JF, Shaw AT, Sequist LV, et al. EGFR mutations and ALK rearrangements are associated with low response rates to PD-1 pathway blockade in non-small cell lung cancer: a retrospective analysis. Clin Cancer Res 2016;22:4585-93. DOI PubMed PMC

13. Ota K, Azuma K, Kawahara A, et al. Induction of PD-L1 expression by the EML4-ALK oncoprotein and downstream signaling pathways in non-small cell lung cancer. Clin Cancer Res 2015;21:4014-21. DOI PubMed

14. Coelho MA, de Carné Trécesson S, Rana S, et al. Oncogenic RAS signaling promotes tumor immunoresistance by stabilizing PD-L1 mRNA. Immunity 2017;47:1083-99.e6. DOI PubMed PMC

15. Assoun S, Theou-Anton N, Nguenang M, et al. Association of TP53 mutations with response and longer survival under immune checkpoint inhibitors in advanced non-small-cell lung cancer. Lung Cancer 2019;132:65-71. DOI PubMed

16. Dong ZY, Zhong WZ, Zhang XC, et al. Potential predictive value of TP53 and KRAS mutation status for response to PD-1 blockade immunotherapy in lung adenocarcinoma. Clin Cancer Res 2017;23:3012-24. DOI PubMed

17. George S, Miao D, Demetri GD, et al. Loss of PTEN is associated with resistance to anti-PD-1 checkpoint blockade therapy in metastatic uterine leiomyosarcoma. Immunity 2017;46:197-204. DOI PubMed PMC

18. Song MS, Salmena L, Pandolfi PP. The functions and regulation of the PTEN tumour suppressor. Nat Rev Mol Cell Biol 2012;13:28396. DOI PubMed

19. Peng W, Chen JQ, Liu C, et al. Loss of PTEN promotes resistance to T cell-mediated immunotherapy. Cancer Discov 2016;6:202-16. DOI PubMed PMC

20. Koyama S, Akbay EA, Li YY, et al. STK11/LKB1 deficiency promotes neutrophil recruitment and proinflammatory cytokine production to suppress T-cell activity in the lung tumor microenvironment. Cancer Res 2016;76:999-1008. DOI PubMed PMC

21. Skoulidis F, Hellmann MD, Awad MM, et al. STK11/LKB1 co-mutations to predict for de novo resistance to PD-1/PD-L1 axis blockade in KRAS-mutant lung adenocarcinoma. JCO 2017;35:9016. DOI

22. Alessi JVM, Glass C, Ricciuti B, Spurr LF, Sholl LM, Awad MM. Clinicopathologic characteristics and immunotherapy outcomes in SMARCA4-mutant (mut) non-small cell lung cancer (NSCLC). JCO 2020;38:9577. DOI

23. Yarchoan M, Hopkins A, Jaffee EM. Tumor mutational burden and response rate to PD-1 inhibition. N Engl J Med 2017;377:2500-1. DOI PubMed PMC

24. Chalmers ZR, Connelly CF, Fabrizio D, et al. Analysis of 100,000 human cancer genomes reveals the landscape of tumor mutational burden. Genome Med 2017;9:34. DOI PubMed PMC

25. Rizvi NA, Hellmann MD, Snyder A, et al. Cancer immunology. Mutational landscape determines sensitivity to PD-1 blockade in nonsmall cell lung cancer. Science 2015;348:124-8. DOI PubMed PMC

26. Le DT, Durham JN, Smith KN, et al. Mismatch repair deficiency predicts response of solid tumors to PD-1 blockade. Science 2017;357:409-13. DOI PubMed PMC

27. Anagnostou V, Smith KN, Forde PM, et al. Evolution of neoantigen landscape during immune checkpoint blockade in non-small cell lung cancer. Cancer Discov 2017;7:264-76. DOI PubMed PMC

28. Hulpke S, Tampé R. The MHC I loading complex: a multitasking machinery in adaptive immunity. Trends Biochem Sci 2013;38:41220. DOI PubMed

29. Gettinger S, Choi J, Hastings K, et al. Impaired HLA class I antigen processing and presentation as a mechanism of acquired resistance to immune checkpoint inhibitors in lung cancer. Cancer Discov 2017;7:1420-35. DOI PubMed PMC

30. He Y, Rozeboom L, Rivard CJ, et al. MHC class II expression in lung cancer. Lung Cancer 2017;112:75-80. DOI PubMed

31. Lastwika KJ, Wilson W 3rd, Li QK, et al. Control of PD-L1 expression by oncogenic activation of the AKT-mTOR pathway in nonsmall cell lung cancer. Cancer Res 2016;76:227-38. DOI PubMed

32. Reck M, Rodríguez-Abreu D, Robinson AG, et al; KEYNOTE-024 Investigators. Pembrolizumab versus chemotherapy for PD-L1positive non-small-cell lung cancer. N Engl J Med 2016;375:1823-33. DOI PubMed

33. Ott PA, Elez E, Hiret S, et al. Pembrolizumab in patients with extensive-stage small-cell lung cancer: results from the phase Ib KEYNOTE-028 study. J Clin Oncol 2017;35:3823-9. DOI PubMed

34. Gao Y, Nihira NT, Bu X, et al. Acetylation-dependent regulation of PD-L1 nuclear translocation dictates the efficacy of anti-PD-1 immunotherapy. Nat Cell Biol 2020;22:1064-75. DOI PubMed PMC

35. Chen X, Pan X, Zhang W, et al. Epigenetic strategies synergize with PD-L1/PD-1 targeted cancer immunotherapies to enhance antitumor responses. Acta Pharm Sin B 2020;10:723-33. DOI PubMed PMC

36. Thomas A, Vilimas R, Trindade C, et al. Durvalumab in combination with olaparib in patients with relapsed SCLC: results from a phase II study. J Thorac Oncol 2019;14:1447-57. DOI PubMed PMC

37. Guo X, Zhang Y, Zheng L, et al. Global characterization of T cells in non-small-cell lung cancer by single-cell sequencing. Nat Med 2018;24:978-85. DOI PubMed

38. Trefny MP, Rothschild SI, Uhlenbrock F, et al. A variant of a killer cell immunoglobulin-like receptor is associated with resistance to PD-1 blockade in lung cancer. Clin Cancer Res 2019;25:3026-34. DOI PubMed

39. Cabrita R, Lauss M, Sanna A, et al. Tertiary lymphoid structures improve immunotherapy and survival in melanoma. Nature 2020;577:561-5. DOI PubMed

40. Helmink BA, Reddy SM, Gao J, et al. B cells and tertiary lymphoid structures promote immunotherapy response. Nature 2020;577:549-55. DOI PubMed

41. Petitprez F, de Reyniès A, Keung EZ, et al. B cells are associated with survival and immunotherapy response in sarcoma. Nature 2020;577:556-60. DOI PubMed

42. Nishikawa H, Sakaguchi S. Regulatory T cells in cancer immunotherapy. Curr Opin Immunol 2014;27:1-7. DOI PubMed

43. Zou W. Regulatory T cells, tumour immunity and immunotherapy. Nat Rev Immunol 2006;6:295-307. DOI PubMed 
44. Chen $\mathrm{C}$, Chen D, Zhang Y, et al. Changes of CD4+CD25+FOXP3+ and CD8+CD28- regulatory $\mathrm{T}$ cells in non-small cell lung cancer patients undergoing surgery. Int Immunopharmacol 2014;18:255-61. DOI PubMed

45. Antony PA, Restifo NP. CD4+CD25+ T regulatory cells, immunotherapy of cancer, and interleukin-2. J Immunother 2005;28:120-8. DOI PubMed PMC

46. Facciabene A, Motz GT, Coukos G. T-regulatory cells: key players in tumor immune escape and angiogenesis. Cancer Res 2012;72:2162-71. DOI PubMed PMC

47. Rech AJ, Vonderheide RH. Clinical use of anti-CD25 antibody daclizumab to enhance immune responses to tumor antigen vaccination by targeting regulatory T cells. Ann N Y Acad Sci 2009;1174:99-106. DOI PubMed

48. Bulliard Y, Jolicoeur R, Zhang J, Dranoff G, Wilson NS, Brogdon JL. OX40 engagement depletes intratumoral Tregs via activating Fc $\gamma$ Rs, leading to antitumor efficacy. Immunol Cell Biol 2014;92:475-80. DOI PubMed

49. Finotello F, Trajanoski Z. New strategies for cancer immunotherapy: targeting regulatory T cells. Genome Med 2017;9:10. DOI PubMed PMC

50. Groth $\mathrm{C}, \mathrm{Hu} \mathrm{X}$, Weber R, et al. Immunosuppression mediated by myeloid-derived suppressor cells (MDSCs) during tumour progression. Br J Cancer 2019;120:16-25. DOI PubMed PMC

51. Huang B, Pan PY, Li Q, et al. Gr-1+CD115+ immature myeloid suppressor cells mediate the development of tumor-induced T regulatory cells and T-cell anergy in tumor-bearing host. Cancer Res 2006;66:1123-31. DOI PubMed

52. Zhao F, Korangy F, Greten TF. Cellular immune suppressor mechanisms in patients with hepatocellular carcinoma. Dig Dis 2012;30:477-82. DOI PubMed PMC

53. Kim K, Skora AD, Li Z, et al. Eradication of metastatic mouse cancers resistant to immune checkpoint blockade by suppression of myeloid-derived cells. Proc Natl Acad Sci U S A 2014;111:11774-9. DOI PubMed PMC

54. De Henau O, Rausch M, Winkler D, et al. Overcoming resistance to checkpoint blockade therapy by targeting PI3K $\gamma$ in myeloid cells. Nature 2016;539:443-7. DOI PubMed PMC

55. Chevolet I, Speeckaert R, Schreuer M, et al. Characterization of the in vivo immune network of IDO, tryptophan metabolism, PD-L1, and CTLA-4 in circulating immune cells in melanoma. Oncoimmunology 2015;4:e982382. DOI PubMed PMC

56. Li A, Barsoumian HB, Schoenhals JE, et al. Indoleamine 2,3-dioxygenase 1 inhibition targets anti-PD1-resistant lung tumors by blocking myeloid-derived suppressor cells. Cancer Lett 2018;431:54-63. DOI PubMed PMC

57. Mantovani A, Sozzani S, Locati M, et al. Macrophage polarization: tumor-associated macrophages as a paradigm for polarized M2 mononuclear phagocytes. Trends Immunol 2002;23:549-55. DOI PubMed

58. Qian BZ, Li J, Zhang H, et al. CCL2 recruits inflammatory monocytes to facilitate breast-tumour metastasis. Nature 2011;475:222-5. DOI PubMed PMC

59. Negus RP, Stamp GW, Relf MG, et al. The detection and localization of monocyte chemoattractant protein-1 (MCP-1) in human ovarian cancer. J Clin Invest 1995;95:2391-6. DOI PubMed PMC

60. Arenberg DA, Keane MP, DiGiovine B, et al. Macrophage infiltration in human non-small-cell lung cancer: the role of CC chemokines. Cancer Immunol Immunother 2000;49:63-70. DOI PubMed

61. Lin EY, Nguyen AV, Russell RG, Pollard JW. Colony-stimulating factor 1 promotes progression of mammary tumors to malignancy. $J$ Exp Med 2001;193:727-40. DOI PubMed PMC

62. Wu Y, Li YY, Matsushima K, Baba T, Mukaida N. CCL3-CCR5 axis regulates intratumoral accumulation of leukocytes and fibroblasts and promotes angiogenesis in murine lung metastasis process. J Immunol 2008;181:6384-93. DOI PubMed

63. Gazzaniga S, Bravo AI, Guglielmotti A, et al. Targeting tumor-associated macrophages and inhibition of MCP-1 reduce angiogenesis and tumor growth in a human melanoma xenograft. J Invest Dermatol 2007;127:2031-41. DOI PubMed

64. Dineen SP, Lynn KD, Holloway SE, et al. Vascular endothelial growth factor receptor 2 mediates macrophage infiltration into orthotopic pancreatic tumors in mice. Cancer Res 2008;68:4340-6. DOI PubMed

65. Ries CH, Cannarile MA, Hoves S, et al. Targeting tumor-associated macrophages with anti-CSF-1R antibody reveals a strategy for cancer therapy. Cancer Cell 2014;25:846-59. DOI PubMed

66. Li X, Wenes M, Romero P, Huang SC, Fendt SM, Ho PC. Navigating metabolic pathways to enhance antitumour immunity and immunotherapy. Nat Rev Clin Oncol 2019;16:425-41. DOI PubMed

67. Tomihara K, Curiel TJ, Zhang B. Optimization of immunotherapy in elderly cancer patients. Crit Rev Oncog 2013;18:573-83. DOI PubMed PMC

68. Kugel CH 3rd, Douglass SM, Webster MR, et al. Age correlates with response to anti-PD1, reflecting age-related differences in intratumoral effector and regulatory T-cell populations. Clin Cancer Res 2018;24:5347-56. DOI PubMed PMC

69. Conforti F, Pala L, Bagnardi V, et al. Cancer immunotherapy efficacy and patients' sex: a systematic review and meta-analysis. Lancet Oncol 2018;19:737-46. DOI PubMed

70. Kichenadasse G, Miners JO, Mangoni AA, Rowland A, Hopkins AM, Sorich MJ. Association between body mass index and overall survival with immune checkpoint inhibitor therapy for advanced non-small cell lung cancer. JAMA Oncol 2020;6:512-8. DOI PubMed PMC

71. Califano R, Kerr K, Morgan RD, et al. Immune checkpoint blockade: a new era for non-small cell lung cancer. Curr Oncol Rep 2016;18:59. DOI PubMed

72. Wang GZ, Zhang L, Zhao XC, et al. The Aryl hydrocarbon receptor mediates tobacco-induced PD-L1 expression and is associated with response to immunotherapy. Nat Commun 2019;10:1125. DOI PubMed PMC

73. Vétizou M, Pitt JM, Daillère R, et al. Anticancer immunotherapy by CTLA-4 blockade relies on the gut microbiota. Science 2015;350:1079-84. DOI PubMed PMC 
74. Routy B, Le Chatelier E, Derosa L, et al. Gut microbiome influences efficacy of PD-1-based immunotherapy against epithelial tumors. Science 2018;359:91-7. DOI PubMed

75. Jin Y, Dong H, Xia L, et al. The diversity of gut microbiome is associated with favorable responses to anti-programmed death 1 immunotherapy in chinese patients with NSCLC. J Thorac Oncol 2019;14:1378-89. DOI PubMed

76. Atarashi K, Tanoue T, Shima T, et al. Induction of colonic regulatory T cells by indigenous Clostridium species. Science 2011;331:337-41. DOI PubMed PMC

77. Gaboriau-Routhiau V, Rakotobe S, Lécuyer E, et al. The key role of segmented filamentous bacteria in the coordinated maturation of gut helper T cell responses. Immunity 2009;31:677-89. DOI PubMed

78. Mazmanian SK, Liu CH, Tzianabos AO, Kasper DL. An immunomodulatory molecule of symbiotic bacteria directs maturation of the host immune system. Cell 2005;122:107-18. DOI PubMed

79. Ready N, Farago AF, de Braud F, et al. Third-line nivolumab monotherapy in recurrent SCLC: CheckMate 032. J Thorac Oncol 2019;14:237-44. DOI PubMed PMC

80. Reck M, Schenker M, Lee KH, et al. Nivolumab plus ipilimumab versus chemotherapy as first-line treatment in advanced non-smallcell lung cancer with high tumour mutational burden: patient-reported outcomes results from the randomised, open-label, phase III CheckMate 227 trial. Eur J Cancer 2019;116:137-47. DOI PubMed

81. Rodriguez-abreu D, Johnson ML, Hussein MA, et al. Primary analysis of a randomized, double-blind, phase II study of the anti-TIGIT antibody tiragolumab (tira) plus atezolizumab (atezo) versus placebo plus atezo as first-line (1L) treatment in patients with PD-L1selected NSCLC (CITYSCAPE). JCO 2020;38:9503-9503. DOI

82. Bracci L, Schiavoni G, Sistigu A, Belardelli F. Immune-based mechanisms of cytotoxic chemotherapy: implications for the design of novel and rationale-based combined treatments against cancer. Cell Death Differ 2014;21:15-25. DOI PubMed PMC

83. Wang Z, Till B, Gao Q. Chemotherapeutic agent-mediated elimination of myeloid-derived suppressor cells. Oncoimmunology 2017;6:e1331807. DOI PubMed PMC

84. Gadgeel SM, Garassino MC, Esteban E, et al. KEYNOTE-189: Updated OS and progression after the next line of therapy (PFS2) with pembrolizumab (pembro) plus chemo with pemetrexed and platinum vs placebo plus chemo for metastatic nonsquamous NSCLC. JCO 2019;37:9013-9013. DOI

85. Reck M, Liu S, Mansfield A, et al. IMpower133: updated overall survival (OS) analysis of first-line (1L) atezolizumab (atezo) + carboplatin + etoposide in extensive-stage SCLC (ES-SCLC). Ann Oncol 2019;30:v710-v711. DOI

86. Socinski MA, Jotte RM, Cappuzzo F, et al; IMpower150 Study Group. Atezolizumab for first-line treatment of metastatic nonsquamous NSCLC. N Engl J Med 2018;378:2288-301. DOI PubMed

87. Neal JW, Lim FL, Felip E, et al. Cabozantinib in combination with atezolizumab in non-small cell lung cancer (NSCLC) patients previously treated with an immune checkpoint inhibitor: results from cohort 7 of the COSMIC-021 study. JCO 2020;38:9610. DOI

88. Gettinger S, Hellmann MD, Chow LQM, et al. Nivolumab plus erlotinib in patients with EGFR-mutant advanced NSCLC. $J$ Thorac Oncol 2018;13:1363-72. DOI PubMed

89. Leal TA, Spira AI, Blakely C, et al. Stage 2 enrollment complete: sitravatinib in combination with nivolumab in NSCLC patients progressing on prior checkpoint inhibitor therapy. Ann Oncol 2018;29:viii400-1. DOI

90. Antonia SJ, Villegas A, Daniel D, et al; PACIFIC Investigators. Durvalumab after chemoradiotherapy in stage III non-small-cell lung cancer. N Engl J Med 2017;377:1919-29. DOI PubMed

91. Theelen WSME, Peulen HMU, Lalezari F, et al. Effect of pembrolizumab after stereotactic body radiotherapy vs pembrolizumab alone on tumor response in patients with advanced non-small cell lung cancer: results of the PEMBRO-RT phase 2 randomized clinical trial. JAMA Oncol 2019;5:1276-82. DOI PubMed PMC

92. Gettinger SN, Wurtz A, Goldberg SB, et al. Clinical features and management of acquired resistance to PD-1 axis inhibitors in 26 patients with advanced non-small cell lung cancer. J Thorac Oncol 2018;13:831-9. DOI PubMed PMC

93. Schoenfeld AJ, Rizvi H, Memon D, et al. Acquired resistance to PD-1 blockade in NSCLC. Ann Oncol 2020;38:9621. DOI

94. Grohé C, Blau W, Gleiber W, et al. Nintedanib+docetaxel in lung adenocarcinoma patients (pts) following treatment with immune checkpoint inhibitors (ICIs): updated efficacy and safety results of the ongoing non-interventional study (NIS) VARGADO (NCT02392455). Ann Oncol 2020;38:9604. DOI 